\title{
MHEALTH: Ferramenta educativa eficaz para controle glicêmico em pacientes diabéticos?
}

\author{
Edith Monielyck Mendonça Batista - UERJ - edithmendonca89@gmail.com - \\ https://orcid.org/0000-0002-3507-5526 \\ Márcia Maria Pereira Rendeiro - UERJ - mmrendeiro@yahoo.com - \\ https://orcid.org/0000-0002-0926-9597
}

\begin{abstract}
Resumo. O objetivo deste estudo é analisar a aplicabilidade da mHealth como instrumento terapêutico educativo para o controle glicêmico de pacientes com Diabetes Mellitus. Para avaliar a eficácia da prática médica no contexto dessa doença crônica foi realizado um levantamento bibliográfico de artigos publicados entre 2010 e 2020 . As análises dos dados permitiram observar um possível potencial da mHealth para ampliar o acesso ao cuidado em saúde, através de práticas educativas. Porém, ainda existe a necessidade de mais estudos que avaliem quais as melhores metodologias a serem utilizadas no processo de ensino-aprendizagem dos pacientes.
\end{abstract}

Palavras- Chaves: Diabetes Mellitus. MHealth. Educação em Saúde. patients?

MHEALTH: Effective educational tool for glycemic control in diabetic

Abstract. The objective of this study is to analyze the applicability of mHealth as an educational tool for therapeutic glycemic control in patients with diabetes mellitus. To assess the effectiveness of medical practice in the context of this chronic disease was based on a literature of articles published between 2010 and 2020. The data analysis allowed to observe a possible potential of mHealth to expand access to health care through educational practices. However, there is still a need for further studies to evaluate what the best methodology to be used in the teaching-learning process of patients.

Keywords: Diabetes Mellitus. MHealth. Health Education.

\section{INTRODUÇÃO}

Há uma expansão das doenças crônicas, onde a Diabetes Mellitus (DM) tem a sua prevalência cada vez maior nas últimas décadas, sendo uma das principais causas de morbimortalidade e uma das quatro principais doenças crônicas com 8,8\% da população mundial, entre 20 e 79 anos de idade, atingida pela doença (CHENG et al., 2013; DIABETES 2019).

A DM é um grupo de doenças metabólicas causadas por alterações no metabolismo da insulina, levando a um estado de hiperglicemia. Ela causa complicações agudas (cetoacidose e a síndrome hiperosmolar hiperglicêmica não cetótica) e complicações crônicas (disfunção renal, vascular, nervosa, ocular) que prejudicam a qualidade de vida do paciente levando a quadros de cegueira, insuficiência renal dialítica, trombose vascular, amputações de membros (BRASIL, 2006; DIABETES 2019).

A redução da incidência e morbidade da DM está relacionada a um melhor acesso a serviços de saúde, com ações voltadas para a educação em saúde e prevenção 
de doenças, visando o autocuidado pelo paciente. $\mathrm{O}$ autocuidado quando ocorre direcionado às necessidades do indivíduo, pode mudar o prognóstico da evolução de uma doença crônica ao promover mudanças comportamentais e um melhor entendimento dos procedimentos empregados para o seu tratamento (SILVA et al., 2009).

A DM é uma doença complexa, onde um dos seus alvos é o controle glicêmico que é medido pela redução do valor da Hemoglobina Glicada-A1c (HbA1c) (DIABETES, 2019). A terapêutica não-farmacológica é essencial para o sucesso na redução da hiperglicemia, onde intervenções educativas tem efeito benéfico no controle glicêmico, adesão ao tratamento medicamentoso, mudanças alimentares e pertencimento pelo paciente ao projeto terapêutico proposto (IQUIZE et al., 2017). Uma das estratégias atuais para aumentar a efetividades dessas ações é a utilização de Tecnologias da Informação e Comunicação (TICs).

$\mathrm{O}$ uso das TICs vem se expandindo com o acesso a dispositivos móveis de comunicação (smartphones, tablet's) como resposta as novas necessidades e anseios de uma população que modificou seu perfil comportamental nas últimas décadas (BESSI et al. 2015). Ligado a esse crescimento, há a criação e lançamentos de softwares para esses dispositivos (conhecidos como app) com diferentes funções.

Sua influência também abrange os aspectos relacionados a saúde, de forma individual e coletiva, pois, o conceito dessa não pode ser dissociado de fatores sociais e econômicos. Os serviços de saúde necessitam se adaptar a essas mudanças para permitir uma maior abrangência das suas ações. Têm-se vários exemplos de app de educação em saúde: CareTaker, Whitebook, Manual MSD, Sanford Guide, Pressão Arterial, entre outros.

Os apps permitem a disponibilização de diversos recursos como, calculadoras e sensores externos e serviços de GPS (Sistema de Posicionamento Global), materiais educativos e criaram um interesse crescente de diferentes setores em Mobile Health (mHealth) em todas as áreas da medicina, tendo usabilidade e resultados promissores em intervenções e educação em saúde (SARDI et al. 2017). Seu uso vem sendo expandido para várias doenças ou situações de saúde, principalmente nas condições crônicas.

Um exemplo da atuação da Saúde Digital em doenças crônicas, tem-se o projeto MyPlan 2.0 que foi uma intervenção de mHealth baseada em Abordagem ao Processo de Ação na Saúde (Health Action Process Approach /HAPA) totalmente automatizada, estruturada através de um site e um aplicativo móvel com intuito de promoção de atividade física (AF), em pacientes com DM e HAS (Hipertensão Arterial Sistêmica). Através do app, os pacientes receberam informações educativas para o estímulo de atividade física. Houve uma diminuição no autorrelato do tempo gasto sentado e aumento na AF moderada a vigorosa (POPPE et al. 2019).

Alguns estudos vêm demonstrando que intervenções de mHealth podem ser uma opção terapêutica, como ferramenta de grande potencial no alcance desse objetivo, promovendo mudanças de hábito de vida nesses indivíduos (CHARPENTIER et al. 2011 e HUO et al. 2019).

O objetivo deste artigo, portanto, é analisar a aplicabilidade da mHealth como instrumento terapêutico educativo para o controle glicêmico de pacientes com DM.

\section{MATERIAL E MÉTODOS}

Este trabalho é um estudo do tipo revisão bibliográfica. A pesquisa bibliográfica foi realizada nas bases de dados PubMed (MEDLINE) e Web of Science em junho de 
2020, com recorte temporal de 2010 a março de 2020. Foram utilizados os seguintes termos traduzidos em língua inglesa: "mHealth", "mobile health," "app," and "digital treatment" de forma combinada com os termos "Diabetes Mellitus". Este estudo teve como pergunta norteadora o seguinte questionamento: qual a influência da mHealth no controle da glicemia e promoção de saúde em pacientes com DM?

Os critérios de inclusão foram estudos clínicos randomizados publicados em língua inglesa que analisassem a aplicabilidade de mHealth na qualidade de vida dos usuários com diagnóstico de DM, tendo como um dos critérios de avaliação a HbA1c; com disponibilidade de texto completo e que avaliassem os resultados da intervenção na vida do usuário. Como critérios de exclusão: artigos que discutiam sobre aspectos econômicos do uso da tecnologia; protocolos de intervenção, revisões sistemáticas e metanálises e artigos que não utilizaram a Telessaúde através de dispositivos móveis ou que tinham como persona: cuidadores ou profissionais de saúde.

Realizou-se, primeiramente, a leitura dos títulos e resumos. Após essa etapa, foi realizada uma pesquisa mais delimitada, onde se objetivou identificar artigos que avaliassem o impacto dessas intervenções na qualidade de vida dos usuários, com ações voltadas para mudanças comportamentais por meio da ampliação do acesso à informações confiáveis. Foram analisados e delimitados, ainda, os resultados com base nos seguintes critérios: foco na promoção de saúde e prevenção de comorbidades; amplitude de atendimento; evidências dos resultados positivos, negativos e aplicabilidade em diferentes localidades. Foi utilizado o checklist presente no Quadro 1 adaptado de MCKAY et al. (2016) para realizar a seleção final dos artigos.

\section{QUADRO 1 - Critérios para avaliação dos estudos}

\begin{tabular}{|l|l|}
\hline $\mathbf{N}^{\mathbf{0}}$ & Questões \\
\hline 1 & O estudo aborda alguma questão (ões) de pesquisa ou Objetivos? \\
\hline 2 & O estudo fornece algum arcabouço teórico para o método de avaliação? \\
\hline 4 & O estudo identifica o país onde a pesquisa foi realizada ? \\
\hline 5 & $\begin{array}{l}\text { O estudo menciona que os aplicativos analisados foram baixados para } \\
\text { avaliação? }\end{array}$ \\
\hline 6 & O estudo fornece uma descrição clara da avaliação e método? \\
\hline 7 & O estudo discute os resultados da avaliação? \\
\hline 8 & O estudo discute alguma limitação? \\
\hline 9 & O estudo fornece alguma recomendação futura em geral? \\
\hline
\end{tabular}

Fonte: adaptado de MCKAY et al. (2016)

\section{RESULTADOS E DISCUSSÃO}

A busca dos artigos realizada nas bases de dados selecionadas resultou em 1.050 artigos dos quais, 467 eram duplicados e foram retirados, restando 583 para a avaliação geral. De tal modo, excluíram-se 406 artigos com base no título e com temáticas não relacionadas ao assunto, restando 35 , lidos na íntegra para avaliar a elegibilidade do estudo. Após a leitura dos 35 artigos, 27 foram excluídos, pois, não contemplam a abordagem qualitativa para a elaboração dos aplicativos, restando 08 para compor a análise deste estudo conforme ilustra a Figura 1. 


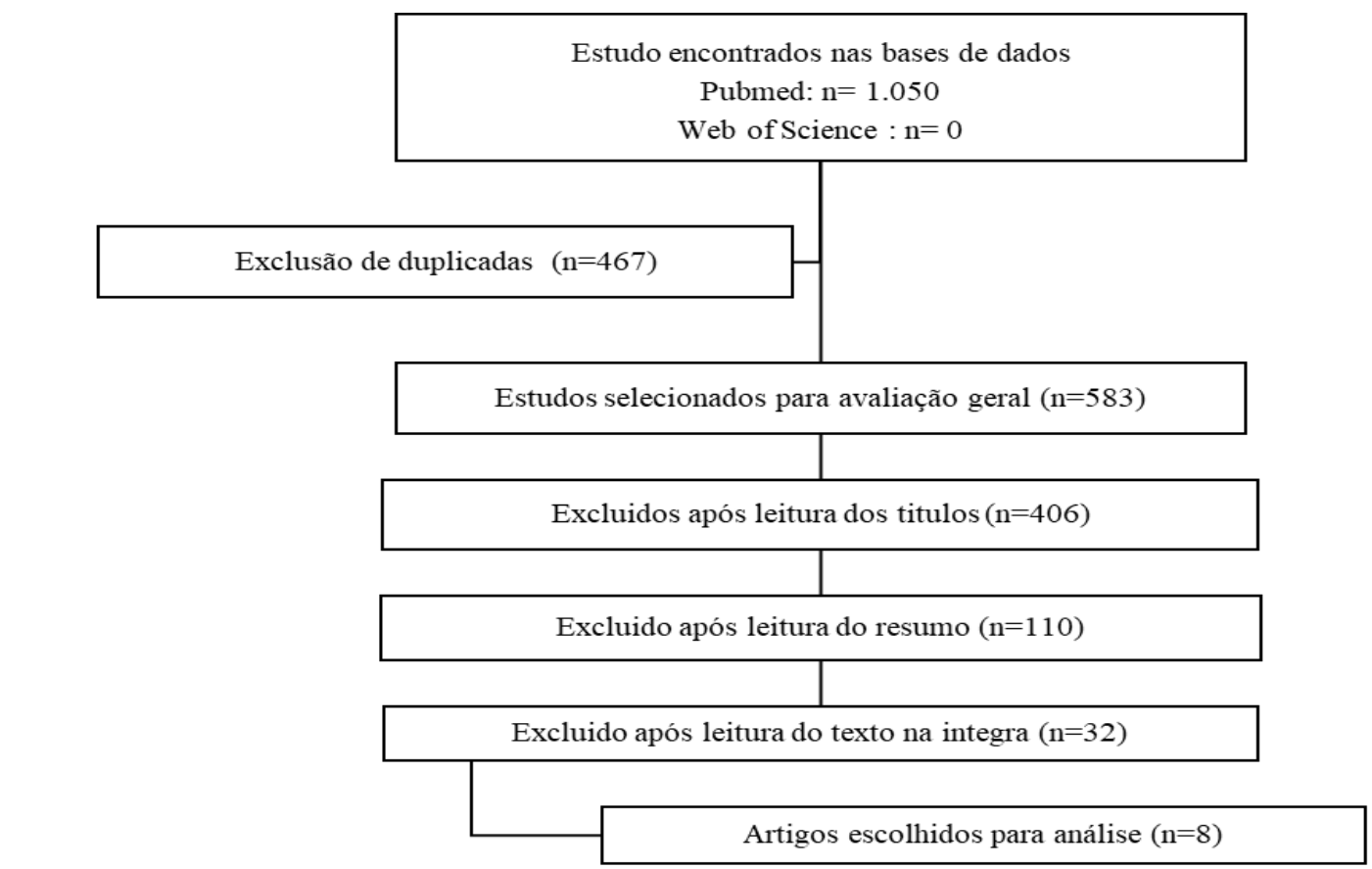

FIGURA 1 - Fluxograma do processo de seleção dos artigos

No Quadro 2, estão representados os 08 artigos selecionados na revisão, organizados como sugerido no estudo de (SARNO et al., 2014). Dos 08 artigos, nos anos de 2011, 2016, 2017 e 2020 obtivemos somente 01 artigo e em 2019, 03 artigos. Houve maior concentração em artigos publicados na Ásia, com 04 artigos. Todos os artigos em língua inglesa e com estudos clínicos randomizados.

\section{QUADRO 2 - Características dos estudos e descrição intervenções em saúde}

\begin{tabular}{|c|c|c|c|}
\hline $\begin{array}{l}\text { Autor e ano } \\
\text { (referência); } \\
\text { país }\end{array}$ & $\begin{array}{l}\text { Delineamento; } \\
\text { duração das } \\
\text { intervenções; } \\
\text { ano de início }\end{array}$ & Descrição das intervenções & $\begin{array}{l}\text { Tecnologia } \\
\text { mHealth; }\end{array}$ \\
\hline $\begin{array}{l}\text { DE ADANA et } \\
\text { al. }(2020), \\
\text { Espanha }\end{array}$ & $\begin{array}{l}\text { Estudo clínico } \\
\text { controlado } \\
\text { randomizado; } 6 \\
\text { meses; } 2014\end{array}$ & $\begin{array}{l}\text { Através do automonitoramento dos níveis } \\
\text { glicêmicos com a interligação entre app e um } \\
\text { glicosímetro gerando gráficos, tabelas, } \\
\text { estatísticas para a terapêutica do DM1 e } \\
\text { disponibilização de informações baseadas nos } \\
\text { resultados apresentados }\end{array}$ & $\begin{array}{l}A p p \text { e versão } \\
\text { web }\end{array}$ \\
\hline $\begin{array}{l}\text { GUO et al. } \\
(2019) \text {, China }\end{array}$ & $\begin{array}{l}\text { Estudo clínico } \\
\text { randomizado, } 13 \\
\text { semanas, } 2015\end{array}$ & $\begin{array}{l}\text { Através do automonitoramento dos níveis } \\
\text { glicêmicos com glicosímetro e inserção dos } \\
\text { dados no app e estímulo ao desenvolvimento de } \\
\text { capacidades de autocuidado }\end{array}$ & $A p p$ \\
\hline $\begin{array}{l}\text { HUO et al } \\
(2019), \text { China }\end{array}$ & $\begin{array}{l}\text { Estudo clínico } \\
\text { randomizado, } 6 \\
\text { meses, } 2016\end{array}$ & $\begin{array}{l}\text { Programa de prevenção secundária baseado em } \\
\text { mensagens de texto com entrega automática } \\
\text { regular com mensagens educativas padronizadas }\end{array}$ & SMS \\
\hline $\begin{array}{l}\text { HUANG et al } \\
(2019), \\
\text { Singapura }\end{array}$ & $\begin{array}{l}\text { Estudo clínico } \\
\text { randomizado, } 12 \\
\text { semanas, } 2018\end{array}$ & $\begin{array}{l}\text { O grupo de intervenção recebeu notas por e-mail } \\
\text { para preencher pesquisas online mensalmente e } \\
\text { uso de app comercial com funções de } \\
\text { agendamento de medicação, lembretes, } \\
\text { rastreamento, compartilhamento de dados, } \\
\text { avaliações de adesão ao tratamento } \\
\text { farmacológico e informações sobre DM }\end{array}$ & $A p p$ e e-mail \\
\hline
\end{tabular}




\begin{tabular}{|c|c|c|c|}
\hline $\begin{array}{l}\text { Autor e ano } \\
\text { (referência); } \\
\text { país }\end{array}$ & $\begin{array}{l}\text { Delineamento; } \\
\text { duração das } \\
\text { intervenções; } \\
\text { ano de início }\end{array}$ & Descrição das intervenções & $\begin{array}{l}\text { Tecnologia } \\
\text { mHealth; }\end{array}$ \\
\hline $\begin{array}{l}\text { KARHULA et } \\
\text { al. } \quad(2015), \\
\text { Finlândia }\end{array}$ & $\begin{array}{l}\text { Estudo clínico } \\
\text { randomizado, } 12 \\
\text { meses, } 2011\end{array}$ & $\begin{array}{l}\text { Ocorreu o automonitoramento dos níveis } \\
\text { glicêmicos e entrega de mensagens educativas e } \\
\text { lembretes com assuntos como alimentação } \\
\text { saudável, práticas de atividades físicas e uso de } \\
\text { medicações. }\end{array}$ & SMS \\
\hline $\begin{array}{l}\text { CHARPENTIER } \\
\text { et al., (2011), } \\
\text { França }\end{array}$ & $\begin{array}{l}\text { Estudo clínico } \\
\text { randomizado, } 6 \\
\text { meses, } 2007\end{array}$ & $\begin{array}{l}\text { O app Diabeo é uma calculadora de bolus de } \\
\text { insulina que permitiu o controle das doses da } \\
\text { medicação a serem aplicada pelos participantes, } \\
\text { como também a realização a contagem de } \\
\text { carboidratos e a atividade física planejada }\end{array}$ & App \\
\hline $\begin{array}{l}\text { KERFOOT et } \\
\text { al., (2017), } \\
\text { Estados Unidos } \\
\text { da América }\end{array}$ & $\begin{array}{l}\text { Estudo clínico } \\
\text { randomizado, } 12 \\
\text { meses, } 2014\end{array}$ & $\begin{array}{l}\text { Houve interação através de pergunta-explicação } \\
\text { de resposta correta com conteúdo sobre controle } \\
\text { da glicose, exercícios, complicações da diabetes } \\
\text { a longo prazo, adesão à medicação e nutrição }\end{array}$ & $\begin{array}{l}A p p-\mathrm{Jogo} \text { e } \\
\text { e-mail }\end{array}$ \\
\hline $\begin{array}{lr}\text { QUINN } & \text { et } \\
(2016), & \text { Estados } \\
\text { Unidos } & \text { da } \\
\text { América } & \end{array}$ & $\begin{array}{l}\text { Estudo clínico } \\
\text { randomizado, } 12 \\
\text { meses }\end{array}$ & $\begin{array}{l}\text { A Intervenção baseou-se no automonitoramento } \\
\text { da glicemia capilar, onde ocorria o registro } \\
\text { desses valores em um app. Além disso, houve o } \\
\text { envio de mensagens educacionais, } \\
\text { comportamentais, motivacionais e específicas } \\
\text { aos dados inseridos }\end{array}$ & App \\
\hline
\end{tabular}

LEGENDA: SMS- Short Message Service

Observa-se um crescimento no número de estudos nos últimos 05 anos sobre o uso de mHealth, em consonância com a expansão do acesso aos smartphones pela população, que vem adotando cada vez mais rapidamente as tecnologias no seu cotidiano, tornando-se um instrumento imprescindível para atividades diárias (SARNO et al. 2014). Essa realidade torna, também, um desafio para a mHealth, pois, devido as constantes atualizações dos diversos modelos de smartphones, pode haver a necessidade de equipes especializadas para uma constante reavaliação dos produtos tecnológicos, gerando um aumento de custo de manutenção.

Quanto a intervenção, 06 artigos abordaram ações voltadas para autocuidado e práticas de monitoramento da glicemia capilar e todos os estudos estimularam a educação em saúde. A educação em saúde permite a construção do conhecimento e formação de multiplicadores que podem estar difundindo na sua comunidade os assuntos aprendidos. Além disso, ao favorecer o aprendizado, ela proporciona alterações no estilo de vida; e também, minimiza as dificuldades encontradas em relação ao conhecimento e atitude dos pacientes (IQUIZE et al., 2017). Há, portanto, o entendimento da necessidade de um autocuidado de qualidade, com comportamentos em consonância com as necessidades individuais e coletivas de cada indivíduo.

O autocuidado possibilita uma "melhoria da saúde percebida, diminuindo ou prevenindo lidar com complicações do DM e diminuindo o custo do cuidado" (SONG 2010, p.95). O desafio da obtenção desse resultado perpassa em entender o processo de tomada de decisão para compreendermos a resposta dos indivíduos perante as ações planejadas em diferentes intervenções.

Kerfoot et al., (2017) elaboraram a interação através de um jogo de perguntas e respostas. Com seu caráter lúdico, os jogos online desinibem, estimulando os usuários a participarem das atividades, sem se sentirem coibidos pelo julgamento de outros integrantes. Além disso, estudos demonstram resultados positivos com o uso de jogos online no cuidado relacionado às doenças crônicas e relacionado à atividade física, promovendo prevenção de doenças e agravos (BRANDÃO et al., 2019). Porém, no estudo 
de Kerfoot et al., (2017), nos pacientes expostos à intervenção não houve diferença quando ao controle glicemia se comparado a ações terapêuticas padrões (QUADRO 3). Criar jogos que consigam realizar a adesão de diferentes públicos deve ter uma abordagem crítica e criativa, focada no problema da persona, com elaboração de uma solução direcionada a necessidade do usuário (CORTES JUNIOR et al., 2020).

Dois artigos usaram a estratégia de SMS (Short Message Service), sem incluir programação de plataforma móvel para interação com os participantes dos estudos. Essa metodologia proporciona que o provedor de saúde relembre constantemente ao indivíduo as práticas de autocuidado, mas percebe-se mais resultados positivos quando o envio das mensagens ocorre de forma personalizada, num feedback constante (HOVADICK, REIS e TORRES, 2019).

No Quadro 3, apresentamos os principais resultados dos artigos quanto ao controle glicêmico, no início e ao final da intervenção. Quanto a população do estudo, todos os estudos analisaram pacientes maiores de 18 anos, sendo 03 publicações direcionadas a pacientes com DM tipo 1 (DM1), 04 com DM tipo 2 (DM2) e 01 com DM gestacional (DMG).

O número de indivíduos estudados variou de 51 a 502 pacientes (QUADRO 3). Quanto ao desfecho redução de HbA1c, 04 estudos demonstraram resultados significativos quanto a diminuição da glicemia dos participantes. Portanto, os artigos trouxeram resultados controversos quanto ao controle glicêmico. Há continuação desse padrão quando estratificamos quanto ao tipo de Diabetes, mantendo a proporção em intervenções com DM1 ou DM2.

A DM é uma doença crônica, resultado de comportamentos alimentares prejudiciais, como dieta rica em carboidratos e gorduras, aliada ao sedentarismo no DM2 e causas genéticas no DM1 (EGEDE et al. 2017). Além disso, mesmo diante do diagnóstico, muitos pacientes não se sentem doentes, principalmente, pelos poucos sintomas apresentados nos anos iniciais da doença (OLIVEIRA et al., 2011).

\section{QUADRO 3 - Principais resultados dos artigos selecionados}

\begin{tabular}{|c|c|c|c|c|c|c|c|}
\hline \multirow[t]{2}{*}{$\begin{array}{l}\text { Autor e ano } \\
\text { (referência) }\end{array}$} & \multirow[t]{2}{*}{$\begin{array}{l}\text { Descrição da } \\
\text { população }\end{array}$} & \multirow[t]{2}{*}{$\begin{array}{c}\text { Amostra } \\
\text { (n) }\end{array}$} & \multicolumn{2}{|c|}{$\begin{array}{c}\text { HbAlc no } \\
\text { tempo } 0(\%), \\
\text { média }\end{array}$} & \multicolumn{2}{|c|}{$\begin{array}{l}\text { HbAlc após } \\
\text { intervenção } \\
(\%)\end{array}$} & \multirow{2}{*}{$\begin{array}{c}\begin{array}{c}\text { Variabilidade } \\
\text { glicêmica }\end{array} \\
\text { P-valor } \\
\end{array}$} \\
\hline & & & GC & GI & GC & GI & \\
\hline $\begin{array}{l}\text { DE ADANA } \\
\text { et al. }(2020)\end{array}$ & $\begin{array}{lr}18-65 \text { anos } & \text { de } \\
\text { idade,> } 2 \text { anos } \\
\text { desde } & 0 \\
\text { diagnóstico } & \text { de } \\
\text { DM1, níveis } & \\
\text { de HbA1c }<8 \%\end{array}$ & 388 & $\begin{array}{l}7,1 \pm \\
0,7\end{array}$ & $\begin{array}{l}7,0 \pm \\
0,6\end{array}$ & $\begin{array}{l}7,0 \pm \\
0,7\end{array}$ & $\begin{array}{l}7,0 \pm \\
0,8\end{array}$ & 0,746 \\
\hline $\begin{array}{l}\text { GUO et al. } \\
\text { (2019) }\end{array}$ & $\begin{array}{l}\text { Gestantes com 21- } \\
45 \text { anos, grávidas } \\
\text { de um único feto e } \\
\text { com DMG entre } \\
24 \text { e } 28 \text { semanas de } \\
\text { gravidez }\end{array}$ & 124 & $\begin{array}{l}5,9 \pm \\
0,3\end{array}$ & $\begin{array}{l}6,0 \pm \\
0,4\end{array}$ & $\begin{array}{l}5,3 \pm \\
0,3\end{array}$ & $\begin{array}{l}4,7 \pm \\
0,2\end{array}$ & $<0,001$ \\
\hline $\begin{array}{l}\text { HUO et al } \\
(2019)\end{array}$ & $\begin{array}{lr}\text { Adultos } & \text { com } \\
\text { diagnóstico } & \text { de } \\
\text { infarto agudo } & \text { do } \\
\text { miocárdio } & \text { ou } \\
\text { intervenção } & \\
\text { coronária } & \\
\text { percutânea) } & \text { e } \\
\text { DM2 } & \end{array}$ & 502 & $\begin{array}{l}7,1 \pm \\
1,4\end{array}$ & $\begin{array}{l}6,9 \pm \\
1,4\end{array}$ & $\begin{array}{l}7,2 \pm \\
1,5\end{array}$ & $\begin{array}{l}6,7 \pm \\
1,3\end{array}$ & 0,003 \\
\hline
\end{tabular}


Conclusão

\begin{tabular}{|c|c|c|c|c|c|c|c|}
\hline \multirow[t]{2}{*}{$\begin{array}{l}\text { Autor e ano } \\
\text { (referência) }\end{array}$} & \multirow[t]{2}{*}{$\begin{array}{l}\text { Descrição } \\
\text { população }\end{array}$} & \multirow[t]{2}{*}{$\begin{array}{l}\text { Amostra } \\
\text { (n) }\end{array}$} & \multicolumn{2}{|c|}{$\begin{array}{l}\text { HbAlc no } \\
\text { tempo } 0(\%) \text {, } \\
\text { média }\end{array}$} & \multicolumn{2}{|c|}{$\begin{array}{l}\text { HbAlc após } \\
\text { intervenção } \\
(\%)\end{array}$} & \multirow{2}{*}{\begin{tabular}{|c|}
$\begin{array}{l}\text { Variabilidade } \\
\text { glicêmica }\end{array}$ \\
P-valor \\
\end{tabular}} \\
\hline & & & $\mathrm{GC}$ & GI & GC & GI & \\
\hline $\begin{array}{l}\text { HUANG et } \\
\text { al (2019) }\end{array}$ & $\begin{array}{l}\text { Pacientes com } \\
\text { DM2, maiores de } \\
21 \text { anos }\end{array}$ & 51 & 8,5 & 8,2 & $\begin{array}{l}9,4 \pm \\
2,4\end{array}$ & $\begin{array}{l}9,0 \pm \\
1,6\end{array}$ & 0,57 \\
\hline $\begin{array}{l}\text { KARHULA } \\
\text { et al., (2015) }\end{array}$ & $\begin{array}{l}\text { Adultos com } \\
\text { doença cardíaca ou } \\
\text { DM2 }\end{array}$ & $\begin{array}{l}250 \\
\text { diabéticos }\end{array}$ & 7,20 & 7,25 & 7,36 & 7,29 & 0,34 \\
\hline $\begin{array}{l}\text { CHARPEN } \\
\text { TIER et al., } \\
2011\end{array}$ & $\begin{array}{l}\text { Pacientes maiores } \\
\text { de } 18 \text { anos com } \\
\text { DM1 e HbAlc > } \\
8 \%\end{array}$ & 180 & $\begin{array}{l}8,91 \\
\pm \\
0,90\end{array}$ & $\begin{array}{l}9,11 \\
\pm \\
1,14\end{array}$ & $\begin{array}{l}9,10 \pm \\
1,16\end{array}$ & $\begin{array}{l}8,63 \pm \\
1,07\end{array}$ & 0,01 \\
\hline $\begin{array}{l}\text { KERFOOT } \\
\text { et al., } 2017\end{array}$ & $\begin{array}{l}\text { Adultos com DM2 } \\
\text { sem controle } \\
\text { glicêmico } \\
\text { adequado }\end{array}$ & 450 & $\begin{array}{l}8,9 \pm \\
3,3\end{array}$ & $\begin{array}{l}9 \pm \\
3,4\end{array}$ & 8,5 & 8,3 & 0,144 \\
\hline $\begin{array}{l}\text { QUINN et } \\
\text { al., } 2016\end{array}$ & $\begin{array}{l}\text { Adultos de } 18 \text { a } 64 \\
\text { anos com DM2, } \\
\text { onde } \\
\text { foram } \\
\text { agrupados quanto } \\
\text { a idade em }>55 \\
\text { anos (G2) e } \leq 55 \\
\text { anos (G1) }\end{array}$ & 163 & $\begin{array}{l}\text { G1: } \\
9,9 \pm \\
1,8 \\
\text { G2: } \\
8,4 \pm \\
1,2\end{array}$ & $\begin{array}{l}\text { G: } \\
19,9 \\
\pm 2,0 \\
\text { G2: } \\
9,8 \pm \\
2,3\end{array}$ & $\begin{array}{l}\text { G1: } \\
8,9 \pm \\
1,9 \\
\text { G2: } \\
8,1 \pm \\
1,5\end{array}$ & $\begin{array}{l}\text { G1: } \\
7,9 \pm \\
1,6 \\
\text { G2: } \\
7,9 \pm \\
1,9\end{array}$ & $\begin{array}{l}\text { G1: } p=0,02 \\
\text { G2: } p=0,001\end{array}$ \\
\hline
\end{tabular}

LEGENDA: GC - Grupo Controle, GI- Grupo Intervenção, G- Grupo

As complicações da doença aparecem a longo prazo, gerando uma falta de motivação do paciente em usar uma plataforma de saúde online e incluir, de forma constante e sustentada, as mudanças que visam a promoção da sua saúde (DU PON et al. 2020). Intervenções educativas a médio prazo trouxeram resultados mais promissores, o que pode estar ligado também a demora em concretizar mudanças comportamentais em prazos mais curtos, necessitando o usuário de um período de adaptação (HOVADICK, REIS e TORRES, 2019).

No DMG há consequências mais mediatas. O feto de mãe com DMG tem maior risco de anomalias congênitas (RIOS et al. 2019), o que gera preocupação para a gestante que promove hábitos saudáveis, mesmo que somente durante a gestação, como foi observado no estudo de Guo et al. (2019).

Intervenções educacionais com indivíduos com DM1 tem ainda outros desafios. Há uma maior incidência de complicações agudas, como a cetoacidose diabéticas, do que em pacientes com DM2, podendo levar a internações e mortalidade. Espera-se um entendimento mais precoce da necessidade de mudanças no seu estilo de vida (DIABETES, 2019). Porém, pacientes com DM1, geralmente, são pacientes mais jovens. O diagnóstico da doença leva a um processo de luto (negação, isolamento, raiva, barganha, depressão e aceitação), onde o doente e os familiares sofrem desgaste físico e emocional (VARGAS et al., 2020). Ao realizarmos uma prática de $m$ Health educativa deve-se entender esse processo e estudos vem demonstrando a necessidade de ações mais periódicas durante um período mais prolongado.

\section{CONCLUSÃO}

A inclusão do uso de mHealth como ferramenta educativa para promoção de saúde, prevenção de doenças e comorbidades em pacientes com doenças crônicas, como DM, é um processo em andamento que vem evoluindo, com mudanças em suas estratégias 
para maior adesão desses usuários às ações propostas por essa prática de cuidado. A Saúde Digital permite a possibilidade de um maior acesso ao cuidado em saúde, porém, ainda temos resultados controversos quanto sua real eficácia e quais as melhores formas de abordagem.

Observa-se resultados positivos com possibilidade de impactos substanciais nas condições de vida dos pacientes com DM quando realizamos ações em educação em saúde aliadas a mHealth, o que permitiria uma interação e maior qualidade em suas decisões de saúde nos usuários, gerando pertencimento aos projetos terapêuticos propostos. Expandir esses resultados para as diversidades de perfis de pacientes e necessidades de cuidados, torna-se um desafio para o desenvolvimento da mHealth. Além disso, fica o questionamento do melhor método para personalizar as metodologias educativas em mHealth.

Ressalta-se a necessidade de mais estudos que avaliem quais as possíveis causas para determinar os fatores que influenciam a fidelização do usuário aos diferentes instrumentos de mHealth e como melhorar a jornada desse cliente ao elaborar o Design Thinking da solução que se busca propor.

\section{REFERÊNCIAS}

BESSI, Vânia Gisele et al. As Tecnologias de Informação e Comunicação e sua Influência na Vivência Espaço-Temporal de Trabalhadores Bancários. Cad.

EBAPE.BR, [s. l.], p. 776-794, 2015.

BRANDÃO, Isabelle De Araújo et al. Revisão Intergrativa Jogos eletrônicos na atenção à saúde de crianças e adolescentes : revisão integrativa. [s. l.], v. 32, n. 4, p. 464-469, 2019.

BRASIL. Cadernos de Atenção Básica - n. ${ }^{0}$ 16: Diabetes Mellitus. - Brasília : Ministério da Saúde, 2006

CHARPENTIER, Guillaume et al. The Diabeo software enabling individualized insulin dose adjustments combined with telemedicine support improves HbA1c in poorly controlled type 1 diabetic patients: a 6-month, randomized, open-label, parallel-group, multicenter trial (TeleDiab 1 Study). Diabetes care, [s. l.], v. 34, n. 3, p. 533-539, 2011.

CHENG, Yiling J. et al. Secular changes in the age-specific prevalence of diabetes among U.S. adults: 1988-2010. Diabetes Care, [s. l.], v. 36, n. 9, p. 2690-2696, 2013.

CÔRTES JÚNIOR, João Carlos de Souza et al. Design Thinking na Reestruturação do Sistema de Avaliação de Disciplina em um Curso de Medicina. Revista Brasileira de Educação Médica, [s. l.], v. 44, n. 4, 2020.

DIABETES, SOCIEDADE BRASILEIRA DE. DIRETRIZES Sociedade Brasileira de Diabetes 2019-2020. [S. l.: s. n.], 2019.

DU PON, Esther et al. Effects of the Proactive Interdisciplinary Self-Management (PRISMA) Program on Online Care Platform Usage in Patients with Type 2 Diabetes in Primary Care: A Randomized Controlled Trial. Journal of diabetes research, [s. l.], v. 2020, p. 5013142, 2020. 
EGEDE, Leonard E et al. Randomized Controlled Trial of Technology-Assisted Case Management in Low Income Adults with Type 2 Diabetes. Diabetes technology \& therapeutics, United States, v. 19, n. 8, p. 476-482, 2017.

GUO, H. et al. Evaluating the effects of mobile health intervention on weight management, glycemic control and pregnancy outcomes in patients with gestational diabetes mellitus. Journal of Endocrinological Investigation, [s. l.], v. 42, n. 6, p. 709-714, 2019.

HOVADICK, Ana Carolina de Andrade; REIS, Ilka Afonso; TORRES, Heloísa Carvalho. Revisão Integrativa autocuidado em DM2 : revisão integrativa. Revisão integrativa, [s. l.], v. 32, n. 2, p. 210-219, 2019.

HUANG, Zhilian et al. A smartphone app to improve medication adherence in patients with type 2 diabetes in Asia: Feasibility randomized controlled trial. JMIR mHealth and uHealth, [s. l.], v. 7, n. 9, 2019.

HUO, Xiqian et al. Effects of Mobile Text Messaging on Glycemic Control in Patients With Coronary Heart Disease and Diabetes Mellitus: A Randomized Clinical Trial. Circulation. Cardiovascular quality and outcomes, United States, v. 12, n. 9, p. e005805, 2019.

IQUIZE, Roxana Claudia Condori et al. Educational practices in diabetic patient and perspective of health professional: a systematic review. Jornal brasileiro de nefrologia, [s. l.], v. 39, n. 2, p. 196-204, 2017.

KARHULA, Tuula et al. Telemonitoring and Mobile Phone-Based Health Coaching Among Finnish Diabetic and Heart Disease Patients: Randomized Controlled Trial. Journal of medical Internet research, [s. l.], v. 17, n. 6, p. e153, 2015.

KERFOOT, B. Price et al. A team-based online game improves blood glucose control in veterans with type 2 diabetes: A randomized controlled trial. Diabetes Care, [s. l.], v. 40, n. 9, p. 1218-1225, 2017.

MCKAY, Fiona H. et al. Evaluating mobile phone applications for health behaviour change: A systematic review. Journal of Telemedicine and Telecare, [s. l.], v. 24, n. 1, p. 22-30, 2016.

OLIVEIRA, Nunila Ferreira de et al. Diabetes Mellitus : desafios relacionados ao autocuidado abor. REBEn, [s. l.], v. 64, n. 2, p. 301-307, 2011.

POPPE, Louise et al. Efficacy of a self-regulation-based electronic and mobile health intervention targeting an active lifestyle in adults having type 2 diabetes and in adults aged 50 years or older: Two randomized controlled trials. Journal of Medical Internet Research, [s. l.], v. 21, n. 8, 2019.

QUINN, Charlene C et al. Mobile Diabetes Intervention for Glycemic Control in 45- to 64-Year-Old Persons With Type 2 Diabetes. Journal of applied gerontology : the official journal of the Southern Gerontological Society, United States, v. 35, n. 2, p. 
227-243, 2016.

RIOS, Washington Luiz Ferreira et al. Repercussões do diabetes mellitus no feto : alterações obstétricas e malformações estruturais. Feminina, [s. l.], v. 45, 2019.

RUIZ DE ADANA, Maria S. et al. Randomized study to evaluate the impact of telemedicine care in patients with type 1 diabetes with multiple doses of insulin and suboptimal HbA1c in Andalusia (Spain): PLATEDIAN study. Diabetes Care, [s. l.], v. 43, n. 2, p. 337-342, 2020.

SARDI, Lamyae; IDRI, Ali; FERNÁNDEZ-ALEMÁN, José Luis. A systematic review of gamification in e-Health. Journal of Biomedical Informatics, [s. l.], v. 71, p. 31-48, 2017.

SARNO, Flavio; SILVA, Daniela; HENRIQUE, Daniel. Mobile health e excesso de peso : uma revisão sistemática. Rev Panam Salud Publica, [s. l.], v. 35, p. 424-431, 2014.

SILVA, Irene de Jesus et al. Cuidado, autocuidado e cuidado de si: uma compreensão paradigmática para o cuidado de enfermagem. Revista da Escola de Enfermagem da USP [online]. v. 43, n. 3, pp. 697-703, 2009.

SONG, Minkyoung. Diabetes Mellitus and the Importance of Self-care. Journal of Cardiovascular Nursing, [s. l.], v. 25, n. 2, p. 93-98, 2010.

VARGAS, Deisi Maria et al. Um Olhar Psicanalítico Sobre Crianças e Adolescentes com Diabetes Mellitus Tipo 1 e seus Familiares. Revista Psicologia e Saúde, [s. l.], v. 12, p. 87-100, 2020. 